\title{
Consumo alimentar e risco cardiovascular em pessoas vivendo com HIV/AIDS
}

\author{
Dietary intake and cardiovascular risk \\ among people living with HIV/AIDS
}

\author{
Luís Fernando Deresz ${ }^{1}$ \\ Carina de Brito ${ }^{2}$ \\ Cláudia Dornelles Schneider ${ }^{3}$ \\ Estela Iraci Rabito ${ }^{4}$ \\ Maria Letícia Rodrigues Ikeda ${ }^{5}$ \\ Pedro Dal Lago ${ }^{1}$
}

\footnotetext{
${ }^{1}$ Programa de PósGraduação em Ciências da Saúde, Universidade Federal de Ciências da Saúde de Porto Alegre (UFCSPA). R. Sarmento Leite 245, Centro. 90050-170 Porto Alegre RS Brasil.lfderesz@gmail.com ${ }^{2}$ Programa de Residência Integrada em Saúde, Grupo Hospitalar Conceição. Porto Alegre RS Brasil.

${ }^{3}$ Programa de PósGraduação em Ciências da Reabilitação, UFCSPA. Porto Alegre RS Brasil.

${ }^{4}$ Departamento de Nutrição, Universidade Federal do Paraná. Curitiba PR Brasil.

${ }^{5}$ Programa de PósGraduação em Saúde Coletiva, Universidade do Vale do Rio dos Sinos. São Leopoldo RS Brasil.
}

\begin{abstract}
This cross-sectional study evaluated the food intake patterns related to cardiovascular risk disease among people living with HIV/AIDS (PLWHA) with viral suppression and receiving highly active antiretroviral therapy (HAART). Food intake was obtained by the annual food frequency questionnaire, separated into two groups, healthy and unhealthy food related intake and cardiovascular disease. Data were analyzed using Student's $t$ Test for independent samples or the Mann-Whitney U Test and Fisher's exact test, with a significance level of $p<0.05$. The sample consisted of 45 individuals with HIV/AIDS (60\% female). The intake of unhealthy foods for cardiovascular risk was greater compared to the intake of healthy foods, both in men $(3.91 \pm 0.26$ vs. $2.79 \pm 0.32 p=0.01)$ and women $(3.40 \pm 0.23 \mathrm{vs}$. $2.60 \pm 0.29 p=0.04)$. Metabolic syndrome prevalence was $33.3 \%$ in men and $37 \%$ in women. Men presented hypertriglyceridemia (50\%) and low HDL (44\%) and women presented central obesity ( $54 \%, p=0.05$ vs. men) hypercholesterolemia $(66.7 \% p=0.02$ vs. men) and hypertriglyceridemia (46\%). Study results indicate the presence of unhealthy food intake patterns and a high prevalence of cardiovascular risk factors in the evaluated subjects.
\end{abstract}

Key words AIDS, Food consumption and cardiovascular diseases
Resumo O presente estudo analisou o consumo alimentar e a presença de fatores de risco cardiovasculares em pessoas vivendo com HIV/AIDS (PVHA) em uso regular de antirretrovirais. $O$ consumo alimentar foi avaliado por meio de questionário de frequência alimentar anual, dividido em alimentos protetores $e$ não protetores para doença cardiovascular (DCV). Os dados foram calculados pelo Teste $t$ de Student para amostras independentes, pelo Teste U de Mann-Whitney e pelo teste exato de Fischer, considerando significativo $p<0,05$. A amostra consistiu de 45 PVHA (60\% do sexo feminino). O consumo de alimentos não protetores para o risco cardiovascular foi maior do que o de alimentos protetores nos homens $(3,91 \pm 0,26$ vs. $2,79 \pm 0,32 p=0,01)$ $e$ nas mulheres $(3,40 \pm 0,23$ vs. $2,60 \pm 0,29 p=$ $0,04)$. A prevalência de síndrome metabólica foi $33,3 \%$ nos homens e $37 \%$ nas mulheres. Os homens apresentaram hipertrigliceridemia (50\%) $e$ baixas concentrações de HDL-c (44\%), enquanto que as mulheres apresentaram hipertrigliceridemia (46\%), hipercolesterolemia $(66,7 \%, p<0,02$ vs. homens) e obesidade central (54\%, $p<0,05$ vs. homens). Os resultados deste estudo indicam a presença de padrão de consumo alimentar inadequado e elevada prevalência de fatores de risco relacionados às DCV nos indivíduos avaliados.

Palavras-chave AIDS, Consumo de alimentos, Doenças cardiovasculares 


\section{Introdução}

Os benefícios do uso regular da terapia antirretroviral combinada (TARV) em relação à diminuição da mortalidade ${ }^{1}$ e melhora na qualidade de vida $^{2}$ estão bem estabelecidos em pessoas vivendo com HIV/AIDS (PVHA). No entanto, em decorrência do uso dos mesmos, existem evidências de efeitos adversos importantes como dislipidemia, intolerância à glicose e hipertensão arterial, todos reconhecidamente fatores de risco para doenças cardiovasculares (DCV) ${ }^{3}$. A combinação destes com o quadro crônico de inflamação, ativação imune e alteração em fatores de coagulação presentes na síndrome potencializa o desenvolvimento das DCV em PVHA ${ }^{4,5}$.

Sabe-se que, independente da infeção pelo HIV, o padrão alimentar apresenta associação com fatores de risco para o desenvolvimento das DCV, e que dietas consideradas pouco saudáveis podem explicar até $30 \%$ dos infartos do miocárdio $^{6,7}$. Por isso, os alimentos passaram a ser classificados em protetores e não protetores para as DCV, conforme sua composição ${ }^{8}$, com o consumo sendo quantificado por ferramentas que fornecem os resultados em escores de frequência. Essa estratégia se mostrou eficaz na avaliação do padrão alimentar de diversas populações ${ }^{8,9}$.

Assim, considerando a importância do consumo alimentar, aliado à carência de estudos na literatura em PVHA, o objetivo deste trabalho foi avaliar o padrão alimentar e a presença de fatores de risco para DCV em PVHA, em um serviço de referência no tratamento do HIV/AIDS na região metropolitana de Porto Alegre, Rio Grande do Sul, Brasil.

\section{Métodos}

\section{Desenho do estudo}

O presente estudo, observacional, foi um recorte transversal, de caráter descritivo-exploratório, com PVHA em uso regular de TARV, selecionados por conveniência, que estavam em acompanhamento no Serviço Especializado em DST/ AIDS Herbert de Souza, Viamão, Rio Grande do Sul, Brasil, entre agosto de 2012 e janeiro de 2014.

\section{Participantes}

Os participantes foram rastreados previamente pela análise do prontuário que seguiu os seguintes critérios de inclusão: homens e mulhe- res com idade entre 18 e 65 anos; sorologia positiva para HIV; uso da mesma TARV há pelo menos seis meses e a carga viral indetectável (utilizado como critério para eficácia da TARV). Foram excluídos do estudo gestantes, lactantes, pacientes sem acompanhamento periódico ambulatorial, ou com duas ou mais faltas consecutivas ao atendimento no Serviço Especializado, pacientes sem exames bioquímicos (validade de um ano contando da data da entrevista), portadores de doença neurológica ou que comprometesse drasticamente a ingestão alimentar, como a anorexia.

\section{Definição do tamanho da amostra}

O cálculo do tamanho da amostra foi obtido considerando o número de pacientes (1300) atendidos no serviço e a estimativa de que $24,4 \%$ apresentassem síndrome metabólica ${ }^{10}$. O erro amostral foi de $10 \%$ e o nível de confiança foi de 90\%, estimando 49 participantes.

\section{Aspectos éticos}

O projeto foi aprovado pelo Comitê de Ética em Pesquisa da Universidade Federal de Ciências da Saúde de Porto Alegre e está em concordância com as normas vigentes da Comissão Nacional de Ética em Pesquisa (466/2012). Todos os participantes do estudo concordaram e assinaram o termo de consentimento livre e esclarecido.

\section{Procedimentos de coleta de dados}

\section{Dados clínicos e exames bioquímicos}

Os dados coletados nos prontuários foram referentes ao último exame realizado. Foram registrados o atual esquema terapêutico, a contagem das células T CD4 e T CD8, da carga viral, do perfil lipídico (lipoproteína de baixa densidade LDL-c, lipoproteína de alta densidade - HDL-c, colesterol total e triglicerídeos) e da glicemia de jejum, desde que realizados até um ano contando da data da entrevista. A frequência de fumantes foi contabilizada por meio de questionamento individualizado sobre o histórico e o consumo, ou não, atual de cigarros.

A presença dos fatores de risco para DCV foi determinada utilizando os critérios do National Cholesterol Education Program - Adult Treatment Panel (NCEP- ATP III) ${ }^{11}$, considerando hipertensão arterial: pressão arterial sistólica $\geq 130 \mathrm{mmHg}$ e/ou pressão arterial diastólica $\geq 85 \mathrm{mmHg}$ e/ou usuário de medicamento anti-hipertensivo; obesidade abdominal: homens $\geq 102 \mathrm{~cm}$, mulheres $\geq$ 
$88 \mathrm{~cm}$; dislipidemia: ser usuário de medicamento hipolipemiante e/ou: triglicerídeos $\geq 150 \mathrm{mg} /$ dl; LDL-c: $\geq 160 \mathrm{mg} / \mathrm{dl}$; HDL-c: homens $\leq 50 \mathrm{mg} /$ $\mathrm{dl}$, mulheres < 40mg/dl; hipercolesterolemia: colesterol total, $\geq 200 \mathrm{mg} / \mathrm{dl}$; glicemia elevada: glicemia de jejum $\geq 110 \mathrm{mg} / \mathrm{dl}$ e/ou usuário de medicamento hipoglicemiante. A presença de três ou mais fatores de risco, com exceção na concentração de LDL-c, foi utilizada para definir síndrome metabólica $(\mathrm{SM})^{11}$ e a estratificação de risco cardiovascular foi realizada pelo escore de Framingham $^{12}$.

\section{Dados antropométricos}

As medidas da massa corporal e estatura foram realizadas em balança mecânica antropométrica (Welmy, modelo R-110, São Paulo), com capacidade máxima de $150 \mathrm{~kg}$ e $200 \mathrm{~cm}$, com precisão de $0,1 \mathrm{~kg}$ e $1 \mathrm{~cm}$, respectivamente. A pesagem do participante foi realizada com o mesmo descalço e com roupas leves. A estatura foi aferida com o participante descalço em pé e com os braços estendidos ao longo do corpo, olhando para a linha do horizonte. Os calcanhares, as nádegas e os ombros estavam alinhados. A medida da circunferência abdominal foi realizada com o paciente em pé, ereto, com os braços ao longo do corpo, com fita antropométrica inelástica e inextensível, no ponto médio entre a borda súpero-lateral da crista ilíaca e o rebordo costal inferior, conforme as orientações da Organização Mundial de Saúde ${ }^{13}$. A pressão arterial, sistólica e diastólica, foi medida pelo método auscultatório após o participante estar sentado por, pelo menos, cinco minutos. A média de três medidas, com intervalo de 5 minutos, foi registrada como valor da pressão arterial.

\section{Consumo alimentar}

O consumo de alimentos foi avaliado por meio de um questionário de frequência alimen$\operatorname{tar}$ (QFA) do tipo quantitativo ${ }^{14}$, que consiste em um checklist de alimentos distribuídos segundo o consumo diário, semanal, quinzenal, mensal, anual ou sazonal ${ }^{15}$. O QFA utilizado foi o desenvolvido para a Região Sul e adaptado para a realidade do estudo referente aos 12 últimos meses ${ }^{14}$. O mesmo foi empregado para mensurar a frequência do consumo de alimentos considerados protetores e não protetores para $\mathrm{DCV}^{12,16}$.

O grupo de alimentos protetores baseou-se nos alimentos de origem vegetal com maior teor de fibras e menor teor energético, enquanto que o grupo de alimentos não protetores incluiu alimentos com lipídios de origem animal, frituras e margarina ${ }^{8,12,16}$. Nenhuma preparação mista foi desagregada, prevalecendo o ingrediente básico de cada preparação. Desta forma os alimentos foram agrupados em:

Alimentos protetores: frutas e sucos naturais; hortaliças (folhosas e não folhosas); leguminosas; e cereais integrais e derivados (arroz, pães, biscoitos salgados e farinhas), somando 20 preparações.

Alimentos não protetores: produtos lácteos integrais (queijos e requeijão); gorduras de origem animal (banha e manteiga); gorduras de origem vegetal (margarinas); alimentos fritos (batata, pastéis e salgadinhos); carnes (aves, peixe frito, bovina e suína), produtos derivados (embutidos, salsicha, hambúrguer e preparações à base de carnes) e ovos; cereais refinados (pães e biscoitos) totalizando 36 preparações.

A frequência do consumo dos alimentos protetores e não protetores foi classificada em baixa (uma até três vezes ao mês), moderada (duas a três vezes na semana) e alta (três a sete vezes na semana).

A avaliação do consumo alimentar ocorreu com os grupos descritos acima, que foram avaliados de acordo com categorias de frequência (S). Foi adotado para cada categoria de frequência um valor ponderado de acordo com o consumo anual de cada alimento, no qual o mínimo correspondeu a alimentos nunca consumidos e o máximo aos consumidos diariamente $(\mathrm{S} 1=0$ alimentos nunca consumidos; S2 = 0,016 alimentos consumido uma ou nenhuma vez por mês; S3 $=0,08$ alimentos consumidos duas ou três vezes por mês; $\mathrm{S} 4=0,2$ alimentos consumidos uma ou duas vezes por semana; S5 $=0,46$ alimentos consumidos três ou quatro vezes por semana; S6 $=0,72$ alimentos consumidos cinco ou seis vezes por semana e S7 = 1 são os alimentos consumidos diariamente $)^{8,9}$. A soma de cada alimento em seu grupo gerou um valor de escore de frequência de consumo, sugerindo que quanto maior o escore, mais o indivíduo consumia daquele grupo. Os parâmetros da Dietary Reference Intakes (DRI) ${ }^{17}$ foram utilizados como valores de referência para os macros e micronutrientes. O QFA, as medidas antropométricas e os valores da pressão arterial foram realizados por uma pesquisadora da equipe com treinamento específico para a função.

\section{Análise estatística}

A análise estatística foi desenvolvida por procedimentos descritivos (frequência, medidas de tendência central e dispersão). Os critérios de 
normalidade foram avaliados pelo teste de Shapiro-Wilk e a comparação entre os grupos (homens vs. mulheres e alimentos protetores vs. não protetores) foi verificada pelo teste $t$ de Student para amostras independentes ou pelo Teste $\mathrm{U}$ de Mann-Whitney, conforme a normalidade dos dados. A distribuição das frequências de valores normais ou alterados para os fatores de risco de DCV entre homens e mulheres foi avaliada pelo teste exato de Fisher. A associação do consumo de alimentos com os fatores de risco para DCV foi avaliada pela correlação de Pearson. O nível de significância considerado foi $\mathrm{p}<0,05$ e as análises foram realizadas no programa estatístico GraphPad Prism 5 for Windows.

\section{Resultados}

Inicialmente foram selecionados 97 indivíduos, destes $48(48,9 \%)$ não compareceram no dia da entrevista, quatro $(4,2 \%)$ se recusaram a participar e $45(46,9 \%)$ foram entrevistados. A média de idade dos participantes foi $42 \pm 10$ anos e 27 (60\%) eram do sexo feminino. As mulheres apresentaram maiores valores de colesterol total $(\mathrm{p}=$ $0,01)$, LDL-c $(p=0,02)$ e HDL-c $(p=0,01)$ na comparação com homens. As características da amostra estão descritas na Tabela 1.

Observou-se baixo risco cardiovascular, pelo escore de Framingham tanto nas mulheres quanto nos homens, com somente três destes sendo classificados com risco intermediário para DCV. Dez mulheres $(37 \%)$ e seis homens $(33,3 \%)$ foram classificados como portadores de síndrome metabólica (35,6\% na amostra total) e não foram verificadas associações entre os fatores de risco e o consumo de alimentos "protetores" e "não protetores" para o risco de DCV. A amostra feminina apresentou maior prevalência de dislipidemia (colesterol total $>200 \mathrm{mg} / \mathrm{dl}, \mathrm{p}=0,01 \mathrm{e}$ LDL-c, $\mathrm{p}$ $=0,02)$ e obesidade abdominal $(\mathrm{p}=0,01)$ quan-

Tabela 1. Caracterização da amostra em pessoas vivendo com HIV/AIDS em uso regular de TARV, Porto Alegre, RS, 2015.

\begin{tabular}{|c|c|c|c|c|}
\hline & Total $n=45$ & Homens $n=18$ & Mulheres n $=27$ & \\
\hline & Média \pm DP & Média \pm DP & Média \pm DP & p-valor \\
\hline \multicolumn{5}{|l|}{ Dados demográficos } \\
\hline Idade (anos) & $42,6 \pm 9,9$ & $39,1 \pm 9,2$ & $44,8 \pm 9,9$ & 0,05 \\
\hline \multicolumn{5}{|l|}{ Dados antropométricos } \\
\hline Estatura $(\mathrm{m})$ & $1,6 \pm 0,1$ & $1,7 \pm 0,1$ & $1,6 \pm 0,1$ & 0,001 \\
\hline Massa corporal (kg) & $67,2 \pm 13,7$ & $72,3 \pm 12,1$ & $63,8 \pm 13,8$ & 0,04 \\
\hline $\operatorname{IMC}\left(\mathrm{kg} / \mathrm{m}^{2}\right)$ & $25,6 \pm 4,5$ & $25,3 \pm 3,6$ & $25,8 \pm 5,0$ & NS \\
\hline $\mathrm{CA}(\mathrm{cm})$ & $88,2 \pm 11,3$ & $88,8 \pm 10,6$ & $87,8 \pm 11,7$ & NS \\
\hline \multicolumn{5}{|l|}{ Dados bioquímicos } \\
\hline Glicemia $(\mathrm{mg} / \mathrm{dL})$ & $92,8 \pm 22,4$ & $90,2 \pm 11,3$ & $94,5 \pm 27,6$ & NS \\
\hline Colesterol Total $(\mathrm{mg} / \mathrm{dL})$ & $201,3 \pm 50,2$ & $176,3 \pm 47,7$ & $218,0 \pm 45,2$ & 0,01 \\
\hline $\mathrm{LDL}-\mathrm{c}(\mathrm{mg} / \mathrm{dL})$ & $122 \pm 44,4$ & $103,6 \pm 36,7$ & $135,0 \pm 45,0$ & 0,02 \\
\hline HDL-c (mg/dL) & $49,7 \pm 19,6$ & $40,8 \pm 13,8$ & $56,3 \pm 20,9$ & 0,01 \\
\hline Triglicerídeos (mg/dL) & $156,7 \pm 74,7$ & $162,4 \pm 77,6$ & $150,7 \pm 74,6$ & NS \\
\hline \multicolumn{5}{|l|}{ Dados clínicos } \\
\hline PAS (mmHg) & $121,2 \pm 15,9$ & $123,4 \pm 13,0$ & $119,7 \pm 17,6$ & NS \\
\hline PAD (mmHg) & $78,6 \pm 11,2$ & $81,5 \pm 9,4$ & $77 \pm 12,1$ & NS \\
\hline T CD4 (céls/m³) & $594 \pm 236$ & $554 \pm 237$ & $621 \pm 237$ & NS \\
\hline T CD8 (céls/m³) & $1115 \pm 676$ & $1317 \pm 935$ & $982 \pm 395$ & NS \\
\hline \multicolumn{5}{|l|}{ TARV - n (\%) } \\
\hline IP + INTR & $24(53,3)$ & $6(33,3)$ & $18(66,7)$ & \\
\hline IP + INTR + INNTR & $12(26,7)$ & $8(44,5)$ & $4(14,8)$ & \\
\hline INTR + INNTR & $9(20)$ & $4(22,2)$ & $5(18,5)$ & \\
\hline
\end{tabular}

Os valores estão expressos em média \pm desvio padrão (DP) ou n (\%); TARV: terapia antirretroviral combinada (dados referentes ao esquema terapêutico utilizado no momento de participação no estudo); IMC: índice de massa corporal, CA: circunferência abdominal, LDL-c: lipoproteína de baixa densidade; HDL-c: lipoproteína de alta densidade; PAS: pressão arterial sistólica; PAD: pressão arterial diastólica; T CD4: linfócitos T CD4; T CD8: linfócitos T CD8; IP: inibidores de protease; INTR: inibidores nucleosídeos da transcriptase reversa; INNTR: inibidores não nucleosídeos da transcriptase reversa; NS: sem diferença estatística. Teste t para amostras independentes para a comparação entre homens e mulheres $(\mathrm{p}<0,05)$. 
do comparado à masculina. Mais informações sobre a presença de fatores de risco para DCV são apresentadas na Tabela 2.

O consumo de alimentos "não protetores" para o risco de DCV em relação aos alimentos "protetores" foi maior tanto na amostra feminina $(3,40 \pm 0,23$ vs. $2,60 \pm 0,29 p=0,04)$ quanto na masculina $(3,91 \pm 0,26$ vs. $2,79 \pm 0,32 \mathrm{p}=0,01)$ (Figura 1). No grupo dos alimentos "não protetores", o pão francês e a margarina foram os que apresentaram alta frequência de consumo, seguido do queijo muçarela classificado como frequência de consumo moderada. Já no grupo de alimentos "protetores" o feijão preto cozido apresentou alta frequência de consumo, seguido por consumo moderado de alface, agrião, rúcula, banana, bergamota e suco natural de laranja. Os dados detalhados do consumo semanal dos alimentos "não protetores" e "protetores" está representado nas Figuras 2A e 2B, respectivamente. No grupo dos alimentos "não protetores" foram consumidos os alimentos: manteiga, frango (frito, grelhado e empanado) sanduíche tipo hambúrguer, polenta frita, almôndega de carne de gado, carne de porco assada, ovos (omelete, ovo mexido e cozido), peixe frito, fígado de gado, requeijão light, torrada industrializada, peixe ensopado, carne seca/carne de sol/charque, mas não foram numericamente representativos e por isso não constam no gráfico apresentado.

A ingestão das vitaminas A, C, E e fibras pelos participantes do estudo apresentou-se abaixo do recomendado ${ }^{17}$ em $85,2,63,88,9$ e $85,2 \%$ das mulheres e em 72,2, 50, 61,1 e 83,3\% dos homens, respectivamente. A descrição dos macro e micronutrientes dos alimentos dos grupos protetores $\mathrm{e}$ não protetores está apresentada na Tabela 3 .

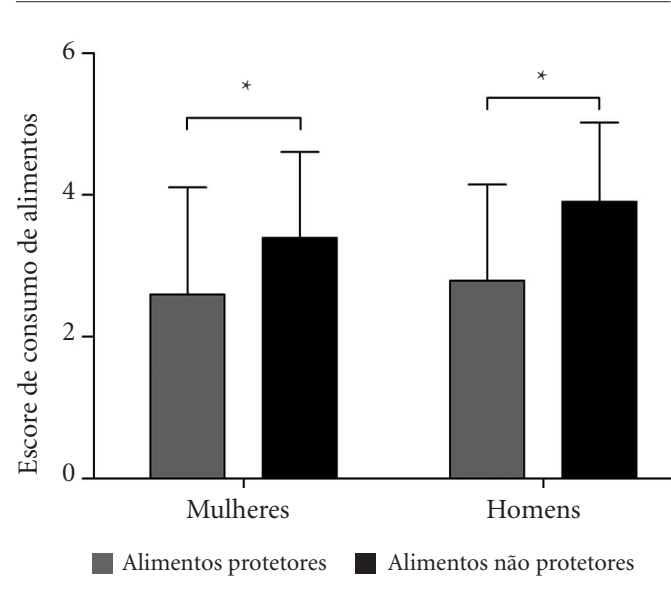

Figura 1. Escores de consumo de alimentos protetores e não protetores em pessoas vivendo com HIV/AIDS em uso regular de TARV, Porto Alegre, RS, 2015.

Valores expressos em média \pm desvio padrão. ${ }^{\star}=\mathrm{p}<0,05$ comparando o consumo de alimentos protetores e não protetores para o risco de doença cardiovascular; análise estatística realizada através do teste $\mathrm{T}$ de Student para amostras independentes.

Tabela 2. Fatores de risco para doença cardiovascular em pessoas vivendo com HIV/AIDS em uso regular de TARV, Porto Alegre, RS, 2015.

\begin{tabular}{|c|c|c|c|c|}
\hline & Total $n=45$ & Homens $n=18$ & Mulheres $\mathbf{n}=27$ & \\
\hline & n (\%) & $\mathbf{n}(\%)$ & n (\%) & p-valor \\
\hline \multicolumn{5}{|l|}{ Dados antropométricos } \\
\hline Obesidade abdominal & $16(35,6)$ & $2(11,2)$ & $14(51,9)$ & 0,01 \\
\hline \multicolumn{5}{|l|}{ Dados clínicos } \\
\hline Hipertensão arterial & $11(24,4)$ & $3(33,3)$ & $8(29,6)$ & NS \\
\hline \multicolumn{5}{|l|}{ Dados bioquímicos } \\
\hline Glicemia elevada & $5(11,1)$ & $3(16,7)$ & $2(7,4)$ & NS \\
\hline Hipercolesterolemia & $23(51,1)$ & $5(27,8)$ & $18(66,7)$ & 0,02 \\
\hline LDL-c elevado & $9(20)$ & $3(16,7)$ & $6(22,2)$ & NS \\
\hline HDL-c baixo & $18(40)$ & $9(50,0)$ & $9(33,3)$ & NS \\
\hline Hipertrigliceridemia & $22(48,9)$ & $9(50,0)$ & $13(48,1)$ & NS \\
\hline Fumantes & $8(17,8)$ & $4(22,2)$ & $4(14,8)$ & NS \\
\hline
\end{tabular}

Dados expressos em número (\%). Valores de referência: Obesidade abdominal: homens $\geq 102 \mathrm{~cm}$, mulheres $\geq 88 \mathrm{~cm}$, hipertensão arterial: pressão arterial sistólica $\geq 130 \mathrm{mmHg}$ e/ou pressão arterial diastólica $\geq 85 \mathrm{mmHg}$ e/ou usuário de medicamento anti-hipertensivo; dislipidemia: ser usuário de medicamento hipolipemiante e/ou: triglicerídeos $>150 \mathrm{mg} / \mathrm{dl}$; lipoproteína de baixa densidade (LDL-c): $>160 \mathrm{mg} / \mathrm{dl}$; lipoproteína de alta densidade (HDL-c): homens $<50 \mathrm{mg} / \mathrm{dl}$, mulheres $<40 \mathrm{mg} / \mathrm{dl}$; hipercolesterolemia: colesterol total, $>200 \mathrm{mg} / \mathrm{dl}$; glicemia elevada: glicemia de jejum $\geq 110 \mathrm{mg} / \mathrm{dl}$ e/ou usuário de medicamento hipoglicemiante. Teste exato de Fischer na comparação entre homens e mulheres $(\mathrm{p}<0,05)$. NS = sem diferença estatística. 

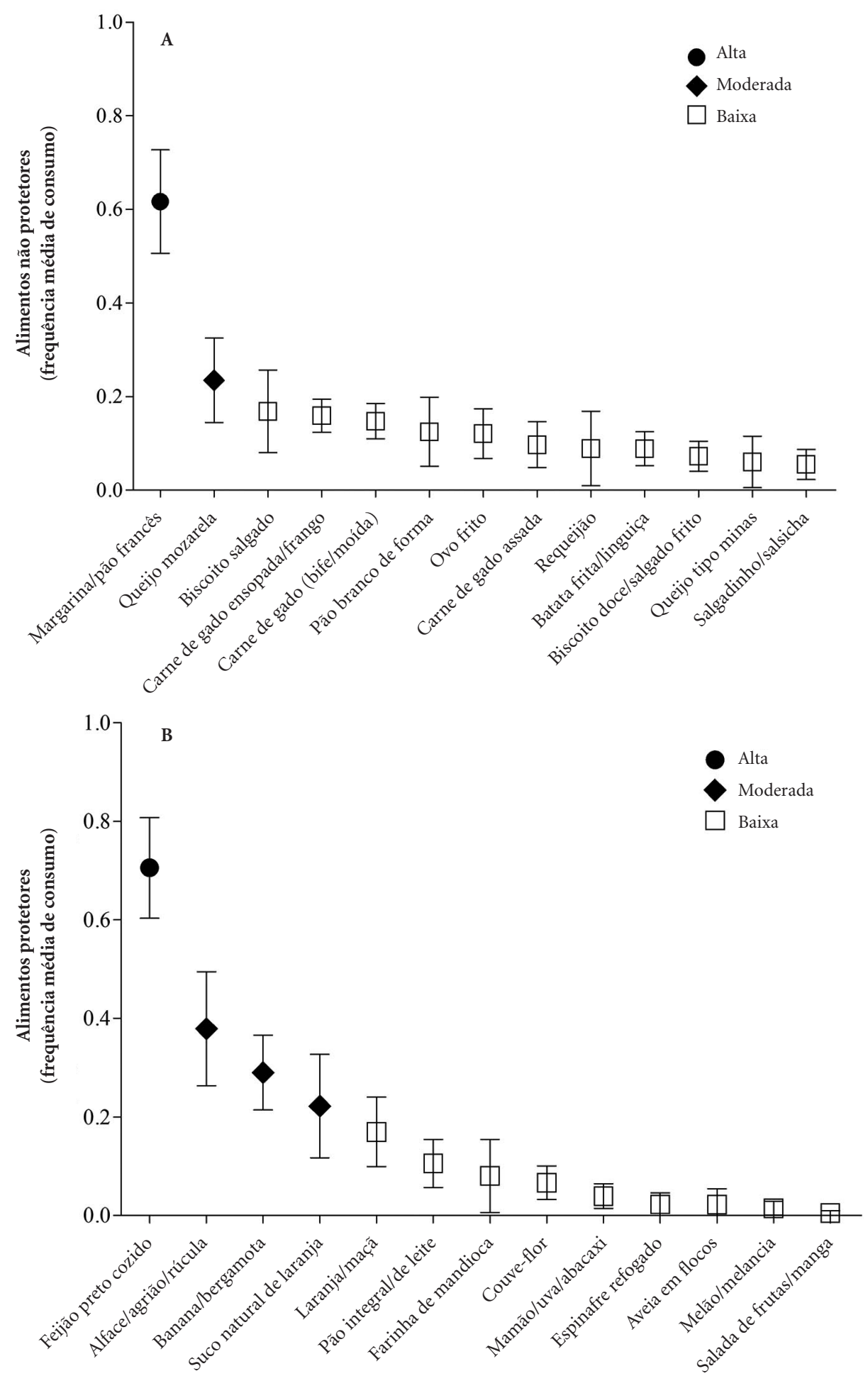

Figura 2. Consumo de alimentos não protetores (A) e protetores (B) para doença cardiovascular em pessoas vivendo com HIV/AIDS, Porto Alegre, RS, 2015.

Os dados estão apresentados em média e intervalo de confiança 95\%. Frequência alta: alimento consumido mais do que três vezes por semana; Moderada: duas a três vezes por semana; Baixa: uma a três vezes por mês. 
Tabela 3. Contribuição de nutrientes conforme o grupo de alimentos protetores e não protetores para doenças cardiovasculares em pessoas vivendo com HIV/AIDS em uso regular de TARV, Porto Alegre, RS, 2015.

\begin{tabular}{lrrrr}
\hline \multicolumn{1}{c}{ Nutriente } & \multicolumn{1}{c}{ Total } & \multicolumn{1}{c}{ Protetores } & \multicolumn{1}{c}{ Não protetores } & \multicolumn{1}{c}{ p-valor } \\
\hline Lipídios $(\mathrm{g})$ & $57,9[9,2-125,1]$ & $7,9[1,7-21,2]$ & $43,6[3,9-110,2]$ & $<0,0001$ \\
Fibras (g) & $13,0[1,2-275,0]$ & $11,2[0,9-60,4]$ & $0,3[0,0-4,0]$ & $<0,0001$ \\
Vitamina A (mg) & $185,4[14,2-8306,7]$ & $43,8[0,7-1050,1]$ & $126,3[24,2-8267,9]$ & $<0,0001$ \\
Vitamina E (mg) & $9,0[1,5-68,6]$ & $3,7[0,4-10,26]$ & $3,9[0,3-24,0]$ & $<0,05$ \\
Vitamina C (mg) & $71,2[1,3-1552,7]$ & $37,5[0,05-1548,7]$ & $3,0[0,05-38,3]$ & $<0,0001$ \\
AGS (g) & $14,7[1,3-37,5]$ & $0,4[0,03-4,6]$ & $14,0[1,6-36,4]$ & $<0,0001$ \\
AGM (g) & $14,7[2,8-35,1]$ & $0,7[0,0-10,1]$ & $13,0[1,1-33,3]$ & $<0,0001$ \\
AGP (g) & $8,6[0,9-226,1]$ & $0,6[0,0-7,7]$ & $7,1[0,3-226,0]$ & $<0,0001$ \\
Colesterol (mg) & $151,4[10,6-542,9]$ & $0,0[0,0-0,0]$ & $151,4[10,6-542,9]$ & $<0,0001$ \\
\hline
\end{tabular}

Os dados apresentados representam à mediana [mínimo e máximo] do conjunto de dados. AGP = Ácido Graxo Poliinsaturado, AGM = Ácido Graxo Monoinsaturado, Teste U de Mann-Whitney na comparação entre alimentos protetores e não protetores $(\mathrm{p}<0,05)$.

\section{Discussão}

Embora seja reconhecida a associação entre o padrão alimentar e o risco de $\mathrm{DCV}^{6,7}$, a melhor compreensão sobre essa temática continua sendo necessária em PVHA. Nesse estudo foi verificado que PVHA apresentam elevada prevalência de SM e possuem padrão alimentar inadequado, configurado pelo maior consumo de alimentos não protetores para $\mathrm{DCV}$ em relação aos protetores. O alto consumo de cereais refinados e ácidos graxos trans, combinados com a baixa ingestão de nutrientes ricos em fibras e minerais (frutas e vegetais), indicam hábitos alimentares que podem contribuir para a exacerbação do risco de DCV presente nessa população.

O padrão alimentar inadequado encontrado nesse estudo reforça os dados da literatura que demonstram dietas pouco saudáveis em PVHA em uso de TARV ${ }^{18-20}$. Nesse sentido, as medidas dietéticas indicadas pela Sociedade Brasileira de Cardiologia (SBC) ${ }^{12}$ e pela American Heart Association (AHA) ${ }^{16}$ para prevenção das DCV e diabetes privilegiam dietas com baixas quantidades de gorduras, carboidratos simples e sódio, e ricas em fibras e sais minerais, formando um padrão alimentar com potencialidade de redução de massa corporal, obesidade abdominal e suavização das anormalidades metabólicas ${ }^{12,16,21}$. Cabe ressaltar que cerca de um terço dos alimentos consumidos no grupo de não protetores (biscoito salgado e doce, linguiça, salsicha, biscoito tipo salgadinho) são caracterizados como ultraprocessados pelo guia alimentar da população brasileira ${ }^{22}$. Portanto, apesar da baixa frequência de consumo, por possuírem predomínio de ingredientes como sal, açúcar, óleos e gorduras, os mesmo contribuem de maneira importante para a ingestão de sódio, ácido graxo saturado e colesterol.

A utilização de dietas com o objetivo de reduzir o risco de DCV em PVHA tem mostrado eficácia tanto na redução na concentração dos triglicerídeos ${ }^{23}$, quanto para minimizar o aumento dos mesmos em decorrência do uso da TARV $^{24}$. Além disso, em contraposição ao resultado observado neste estudo, o uso de dietas ricas em fibras apresenta associação negativa com a deposição de gordura corporal, indicando que a ingestão de fibras em quantidades adequadas é uma estratégia dietética eficaz para a redução do risco de desenvolvimento de $\mathrm{DCV}^{25}$. Entretanto, o acompanhamento nutricional em longo prazo se faz necessário para a manutenção dos benefícios do mesmo, pois a orientação nutricional durante períodos relativamente curtos, até seis meses, não mostrou benefícios nos parâmetros bioquímicos, mas promoveu alterações antropométricas, como prevenção da redistribuição da gordura e ganho de massa corporal ${ }^{26}$.

Concomitantemente, foi identificado neste estudo que o consumo das vitaminas A, C e E apresentavam-se abaixo das recomendações da $\mathrm{DR}^{17} \mathrm{em}$ grande parte da amostra estudada. A deficiência de micronutrientes é uma característica comum em PVHA, podendo ser causada pela combinação da reduzida ingestão de nutrientes, má absorção intestinal e aumentada demanda metabólica ${ }^{27}$. Essas alterações são importantes, pois essas vitaminas são essenciais para o adequado funcionamento do sistema imunológico, além do que já foi verificado que baixos níveis das mesmas estão associados com maior progressão da doença ${ }^{28}$ e elevada taxa de mortalidade ${ }^{29}$ nessa população. Além disso, o baixo consumo das vi- 
taminas A, C e E, consideradas antioxidantes por protegerem as membranas celulares das ações dos radicais livres, está associado com maior risco de DCV $^{30,31}$, reforçando a necessidade de alimentação balanceada nesta população.

Com relação à $\mathrm{SM}$, a prevalência em nossa amostra foi $35,6 \%$, maior do que a observada por Alencastro et al. (17,2 e 24,4\%) em estudos realizados na mesma região ${ }^{10,32}$. A maior prevalência de SM neste estudo pode ser explicada, ainda que parcialmente, pelo uso de TARV, pois, diferentemente dos trabalhos de Alencastro et al. ${ }^{10,32}$, todos os participantes dessa pesquisa estavam realizando o tratamento com TARV, aspecto que reconhecidamente tem propiciado elevação na concentração do colesterol total e trigliceríde$\mathrm{os}^{33}$. Corroborando essas informações, o quadro de SM foi impulsionado, principalmente, pelos altos índices do colesterol total, dos triglicerídeos e pela presença de obesidade abdominal nas mulheres, enquanto que nos homens foi, especialmente, devido à hipertrigliceridemia e ao baixo HDL-c, concordando com quadro verificado pelo estudo de Alencastro et al..$^{32}$, porém, diferente de outro norte-americano ${ }^{34}$, no qual PVHA apresentavam obesidade abdominal e hipertensão arterial como fatores de risco mais presentes para a definição da SM. A diferença observada entre os estudos poder ser explicada, ao menos parcialmente, pelas características socioeconômicas e culturais relacionadas ao padrão alimentar nos distintos países. A presença desses fatores de risco, neste estudo, em especial a hipertrigliceridemia, indica a necessidade de estratégias farmacológicas e não farmacológicas para tratar e preveni-los, no qual a orientação nutricional pode ser uma ferramenta eficaz, conforme mencionado anteriormente ${ }^{23,24}$.
O baixo risco de DCV indicado pelo escore de Framingham encontrado em nossa amostra corrobora dados da literatura ${ }^{35}$. O mesmo pode ser justificado pela menor acurácia dos tradicionais escores de risco para DCV em PVHA ${ }^{36}$. Isso se deve ao fato da etiologia das DCV no HIV/AIDS também estarem associadas a fatores de risco não tradicionais, como a inflamação crônica e a ativação imune, as quais contribuem substancialmente no processo aterosclerótico ${ }^{4,36} \mathrm{e}$, consequentemente, no risco cardiovascular.

As limitações apresentadas no presente estudo estão relacionadas ao tamanho amostral, que pode não refletir a população completa do serviço onde os participantes foram recrutados; à fidedignidade dos exames clínicos, pois em alguns casos os mesmos foram realizados em até um ano antes da execução do estudo; e, ao método de frequência de consumo alimentar aplicado, que pode limitar a avaliação do consumo alimentar da população investigada e subestimar as ingestões de micronutrientes como vitamina A, C e E. Reconhecemos, também, que o uso de variações de TARV por períodos distintos podem promover efeitos colaterais diferentes nos fatores de risco para DCV. E, por fim, acreditamos que a quantificação dos dados referentes ao consumo de álcool e aos níveis de atividade física poderia enriquecer os relativos ao risco cardiovascular da população investigada.

\section{Conclusões}

Os resultados deste estudo indicam a presença de consumo alimentar inadequado e alta prevalência de fatores de risco relacionados ao desenvolvimento e/ou progressão das DCV em PVHA em uso de TARV, reforçando a necessidade de cuidados relacionados a esses desfechos nessa população. 


\section{Colaboradores}

LF Deresz e C Brito participaram da elaboração do projeto de pesquisa, realizaram a coleta, análise e discussão dos dados e redação do manuscrito, considerando ambos como primeiros autores. CD Schneider participou da elaboração do projeto de pesquisa, realizou a análise e discussão dos dados e redação do manuscrito. EI Rabito participou da elaboração do projeto de pesquisa, realizou a análise e discussão dos dados e revisou a versão final do manuscrito. MLR Ikeda participou da coleta dos dados, discussão dos resultados e redação do manuscrito. P Dal Lago participou da elaboração do projeto de pesquisa, redação e revisão da versão final do manuscrito.

\section{Referências}

1. Poorolajal J, Hooshmand E, Mahjub H, Esmailnasab N, Jenabi E. Survival rate of AIDS disease and mortality in HIV-infected patients: a meta-analysis. Public Health 2016; 139:3-12.

2. Passos SM, Souza LD. An evaluation of quality of life and its determinants among people living with HIV/ AIDS from Southern Brazil. Cad Saude Publica 2015; 31(4):800-814.

3. Metkus Junior TS, Brown TT, Post WS. Cardiovascular disease associated with the human immunodeficiency virus: an update. Curr Treat Options Cardiovasc Med 2014; 16(11):346.

4. tein $\mathrm{JH}$, Hsue PY. Inflammation, immune activation, and CVD risk in individuals with HIV infection. JAMA 2012; 308(4):405-406.

5. Erlandson KM, Campbell TB. Inflammation in Chronic HIV Infection: What Can We Do? J Infect Dis 2015; 212(3):339-342.

6. Santos RD, Gagliardi AC, Xavier HT, Magnoni CD, Cassani R, Lottenberg AM, Casella Filho A, Araújo DB, Cesena FY, Alves RJ, Fenelon G, Nishioka SAD, Faludi AA, Geloneze B, Scherr C, Kovacs C, Tomazzela C, Carla C, Barrera-Arellano D, Cintra D, Quintão E, Nakandakare ER, Fonseca FAH, Pimentel I, Santos JE, Bertolami MC, Rogero M, Izar MC, Nakasato M, Damasceno NRT, Maranhão R, Cassani RSL, Perim R, Ramos S. I Diretriz sobre o Consumo de Gorduras e Saúde Cardiovascular. Arq Bras Cardiol 2013; 100(Supl. 3):1-40.

7. Iqbal R, Anand S, Ounpuu S, Islam S, Zhang X, Rangarajan S, Chifamba J, Al-Hinai A, Keltai M, Yusuf S; INTERHEART Study Investigators. Dietary patterns and the risk of acute myocardial infarction in 52 countries: results of the INTERHEART study. Circulation 2008; 118(19):1929-1937.

8. Fornes NS, Martins IS, Velasquez-Melendez G, Latorre MR. Escores de consumo alimentar e níveis lipêmicos em população de São Paulo, Brasil. Rev Saude Publica 2002; 36(1):12-18.

9. Silva EF, Lewi DS, Vedovato GM, Garcia VR, Tenore SB, Bassichetto KC. Nutritional and clinical status, and dietary patterns of people living with HIV/AIDS in ambulatory care in Sao Paulo, Brazil. Rev Bras Epidemiol 2010; 13(4):677-688.

10. Alencastro PR, Fuchs SC, Wolff FH, Ikeda ML, Brandão $\mathrm{AB}$, Barcellos NT. Independent predictors of metabolic syndrome in HIV-infected patients. AIDS Patient Care STDS 2011; 25(11):627-634.

11. National Cholesterol Education Program (NCEP) Expert Panel on Detection Ea, and Treatment of High Blood Cholesterol in Adults (Adult Treatment Panel III). Third Report of the National Cholesterol Education Program (NCEP) Expert Panel on Detection, Evaluation, and Treatment of High Blood Cholesterol in Adults (Adult Treatment Panel III) final report. Circulation 2002; 106(25):3143-3421.

12. Sposito AC, Caramelli B, Fonseca FA, Bertolami MC. IV Diretriz Brasileira Sobre Dislipidemias e Prevenção da Aterosclerose Departamento de Aterosclerose da Sociedade Brasileira de Cardiologia. Arq Bras Cardiol 2007; 88(Supl. 1):2-19.

13. World Health Organization (WHO). Physical Status: The use and interpretation of anthropometry: Report of a WHO Expert Committee. Geneva: WHO; 1995. 
14. Zanolla AF, Olinto MT, Henn RL, Wahrlich V, Anjos LA. Avaliação de reprodutibilidade e validade de um questionário de freqüência alimentar em adultos residentes em Porto Alegre, Rio Grande do Sul, Brasil. Cad Saude Publica 2009; 25(4):840-848.

15. Ribeiro AC, Sávio KEO, Rodrigues MLCF, Costa THM, Schmitz BAS. Validation of a food frequency questionnaire for the adult population. Rev Nutr 2006; 19(5):553-562.

16. American Heart Association Nutrition Committee, Lichtenstein AH, Appel LJ, Brands M, Carnethon M, Daniels S, Franch HA, Franklin B, Kris-Etherton P, Harris WS, Howard B, Karanja N, Lefevre M, Rudel L, Sacks F, Van Horn L, Winston M, Wylie-Rosett J. Diet and lifestyle recommendations revision 2006: a scientific statement from the American Heart Association Nutrition Committee. Circulation 2006; 114(1):82-96.

17. Otten J, Hellwig J, Meyers L. Dietary Reference Intakes: The essential guide to nutrient requirements. Washington: Institute of Medicine of the National Academies; 2005.

18. Duran AC, Almeida LB, Segurado AA, Jaime PC. Diet quality of persons living with HIV/AIDS on highly active antiretroviral therapy. J Hum Nutr Diet 2008; 21(4):346-350.

19. Arendt BM, Aghdassi E, Mohammed SS, Fung LY, Jalali P, Salit IE, Allard JP. Dietary intake and physical activity in a Canadian population sample of male patients with HIV infection and metabolic abnormalities. Curr HIV Res 2008; 6(1):82-90.

20. Hendricks KM, Barrentine JE, Houser RF, Wanke CA. Adherence to the American heart association dietary guidelines by individuals living with HIV infection. $J$ Am Diet Assoc 2006; 106:A30.

21. Lundgren JD, Battegay M, Behrens G, De Wit S, Guaraldi G, Katlama C, Martinez E, Nair D, Powderly WG, Reiss P, Sutinen J, Vigano A; EACS Executive Committee. European AIDS Clinical Society (EACS) guidelines on the prevention and management of metabolic diseases in HIV. HIV Med 2008; 9(2):72-81.

22. Brasil. Ministério da Saúde (MS). Guia alimentar da população brasileira. Brasília: MS; 2014.

23. Stradling C, Chen YF, Russell T, Connock M, Thomas GN, Taheri S. The effects of dietary intervention on HIV dyslipidaemia: a systematic review and meta-analysis. PLoS One 2012; 7:e38121.

24. Lazzaretti RK, Kuhmmer R, Sprinz E, Polanczyk CA, Ribeiro JP. Dietary intervention prevents dyslipidemia associated with highly active antiretroviral therapy in human immunodeficiency virus type 1-infected individuals: a randomized trial. J Am Coll Cardiol 2012; 59(11):979-988.

25. Hadigan C. Dietary habits and their association with metabolic abnormalities in human immunodeficiency virus-related lipodystrophy. Clin Infect Dis 2003; 37(Supl. 2):S101-S104.
26. Fitch K, Abbara S, Lee H, Stavrou E, Sacks R, Michel T, Hemphill L, Torriani M, Grinspoon S. Effects of lifestyle modification and metformin on atherosclerotic indices among HIV-infected patients with the metabolic syndrome. AIDS 2012; 26(5):587-597.

27. Drain PK, Kupka R, Mugusi F, Fawzi WW. Micronutrients in HIV-positive persons receiving highly active antiretroviral therapy. Am J Clin Nutr 2007; 85(2):333345.

28. Tang AM, Graham NM, Semba RD, Saah AJ. Association between serum vitamin A and E levels and HIV-1 disease progression. Aids 1997; 11(5):613-620.

29. Semba RD, Graham NM, Caiaffa WT, Margolick JB, Clement L, Vlahov D. Increased mortality associated with vitamin A deficiency during human immunodeficiency virus type 1 infection. Arch Intern Med 1993; 153(18):2149-2154.

30. Stampfer MJ, Hennekens CH, Manson JE, Colditz GA, Rosner B, Willett WC. Vitamin E consumption and the risk of coronary disease in women. N Engl J Med 1993; 328(20):1444-1449.

31. Rimm EB, Stampfer MJ, Ascherio A, Giovannucci E, Colditz GA, Willett WC. Vitamin E consumption and the risk of coronary heart disease in men. $N$ Engl J Med 1993; 328(20):1450-1456.

32. Alencastro PR, Wolff FH, Oliveira RR, Ikeda ML, Barcellos NT, Brandão AB, Fuchs SC. Metabolic syndrome and population attributable risk among HIV/AIDS patients: comparison between NCEP-ATPIII, IDF and AHA/NHLBI definitions. AIDS Res Ther 2012; 9(1):29.

33. Feeney ER, Mallon PW. HIV and HAART-Associated Dyslipidemia. Open Cardiovasc Med J 2011; 5:49-63.

34. Tiozzo E, Konefal J, Adwan S, Martinez LA, Villabona J, Lopez J, Cutrono S, Mehdi SM, Rodriguez A, Woolger JM, Lewis JE. A cross-sectional assessment of metabolic syndrome in HIV-infected people of low socio-economic status receiving antiretroviral therapy. Diabetol Metab Syndr 2015; 7:15.

35. Pirš M, Jug B, Eržen B, Šabović M, Karner P, Poljak M, Tomažič J. Cardiovascular risk assessment in HIV-infected male patients: a comparison of Framingham, SCORE, PROCAM and DAD risk equations. Acta Dermatovenerol Alp Pannonica Adriat 2014; 23(3):43-47.

36. D'Agostino RB. Cardiovascular risk estimation in 2012: lessons learned and applicability to the HIV population. J Infect Dis. 2012; 205(Supl. 3):S362-S367.

Artigo apresentado em 15/12/2015

Aprovado em 05/09/2016

Versão final apresentada em 07/09/2016 